\title{
Effect of Intratympanic Dexamethasone in Idiopathic Sudden Sensorineural Hearing Loss
}

\section{Rabi Hembrom, ${ }^{1}$ Indranil Sen, ${ }^{1}$ Bina Tamang, ${ }^{1}$ Satadal Mandal, ${ }^{1}$ Amit Chakrabarti ${ }^{1}$}

\section{Introduction:}

\section{ABSTRACT}

Oral steroids currently represent the standard treatment for idiopathic sudden sensorineural hearing loss The aim of this study is to assess the effectiveness of intratympanic dexamethasone injection for treating 'Idiopathic sudden sensori-neural hearing loss' (ISSNHL) not improved with conventional oral steroid.

\section{Materials and Methods:}

A prospective study was conducted on 30 patients refractory to oral steroid therapy between June 2017 to May 2018. 'Intratympanic dexamethasone injection' (ITDI) was given every week for 3 consecutive weeks. Hearing was assessed by performing pure tone audiogram before every ITDI and also 1 week after the completion of treatment.

Results:

Hearing improvement was found in 19 out of the 30 cases (63.3\%).

Conclusions:

Intratympanic dexamethasone significantly improves the prognosis of ISSNHL and is a safe, inexpensive and effective treatment. Keywords:

Hearing Loss, Sudden; Hearing Loss, Sensorineural; Dexamethasone, Intratympanic

$\mathrm{S}$ udden sensorineural hearing loss (SSNHL) is defined as a hearing loss of $30 \mathrm{~dB}$ or more, over at least three contiguous audiometric frequencies, that develops over 72 hours or less. ${ }^{1}$ The incidence of SSNHL is very low, approximately five to 20 per 100,000 population per year., ${ }^{2,3}$ Although this disease is still considered idiopathic, many authors have suggested viral infections, vascular insufficiency, and immunological or inflammatory conditions as possible aetiologies. It is a devastating disease for patients and represents a true otologic emergency. There are many etiologies of sudden hearing loss, but only about $10-15 \%$ of cases have an identifiable cause. $^{4}$

Oral steroids have been effective in many patients and currently represent the standard treatment for idiopathic sudden sensorineural hearing loss (ISSNHL). ${ }^{5}$ Steroids

1 - Midnapore Medical College, Midnapore

Corresponding author:

Dr Bina Tamang

email: tbina39@gmail.com are believed to reduce inner ear inflammation and autoimmune response and to be beneficial for recovery of nerve function. Steroid receptors have been found in the inner ear and may explain why steroid therapy is effective. But for patients with diabetes, tumors, peptic-ulcers, tuberculosis, hypertension and other systemic disorders, steroid therapy may not be appropriate. Even in those patients where oral steroids are not contraindicated, the failure rate is around one third. ${ }^{6}$ Intratympanic steroids injection is a new treatment choice for these patients, and may also offer alternatives for cases that have failed to respond to typical medical treatments. Intratympanic steroids (ITSs) may treat ISSNHL more effectively than oral steroids. ${ }^{7}$

Data to support this hypothesis are limited almost exclusively to case series, which differ in the type of steroid used, the steroid dose, the dose frequency, the method of injection, the existence of previous treatment with oral steroids, and the outcome measurements. Most cases of sudden hearing loss are idiopathic not because they do not have a cause but because we are unable to 
identify it.

Steroid therapy has been widely used as the empirical treatment for various inner ear diseases such as sudden sensorineural hearing loss, vertigo in Menieres disease, and autoimmune induced hearing loss for a long time.

Two major lines of evidence support the feasibility of intratympanic steroid injection treatment and explaining its mechanism of action. First, injected steroid into the middle ear cavity can penetrate the round window membrane and diffuse into the inner ear fluid. Second, many glucocorticoid and mineralocorticoid receptors have been found in the inner ear structure. ${ }^{8}$

\section{Materials and Methods}

It was a prospective study conducted on 30 patients in the age group of 25 to 70 years, diagnosed with unilateral SSNHL of unknown causes attending the out-patient department of ear, nose and throat at peripheral tertiary care hospital. The study was conducted from June 2017 to May 2018. Ethical clearance was obtained from the institute's ethical clearance committee. Informed consent was taken from the cases after explaining the procedure. For this study we selected patients of hearing loss, who fulfill the definition of SSNHL, who had been previously treated with oral steroids for 7 days and were found non- responsive, and those who presented with their symptoms within one month of onset. Patients with somatic pathology such as hypertension, diabetes mellitus, T.B., glaucoma etc where systemic steroids were strictly contraindicated were excluded. Also cases with autoimmune diseases, oncology patients, acoustic neuroma, cases with history of intake of ototoxic drugs, pregnant and nursing women and cases with middle ear pathology were excluded from the study. Cases with SSNHL in the only hearing ear were also excluded.

All the patients were examined and relevant investigations done including pure tone audiogram. All the patients included in the study received an initial therapy of oral steroid with prednisolone (1-2 mg/kg/ day, maximum $60 \mathrm{mg} /$ day) for 7 days. Patients who failed to recover after initial oral steroid therapy were given three intratympanic injection of dexamethasone.

\section{Method of intratympanic injection:}

The patient is positioned in the supine position with head tilted 45 degrees to the opposite side. With a $1 \mathrm{ml}$ syringe and spinal needle, a puncture was made in the posteroinferior quadrant of tympanic membrane, $0.5-1$ $\mathrm{ml}$ of dexamethasone injection $(4 \mathrm{mg} / \mathrm{ml})$ was injected through this site. Intratympanic dexamethasone injection was given once a week for 3 consecutive weeks.

Patients were evaluated for pure-tone thresholds both before and after steroid injection. The pure-tone average (PTA) was calculated as an average of thresholds measured at 0.5, 1.0 and $2.0 \mathrm{KHz}$.

Data was analyzed by using mean, standard deviation and Paired t- test. Improvement was considered when pure tone average return to within $10 \mathrm{~dB}$ HL of unaffected ear. ${ }^{1,9}$

\section{Results}

Total 30 diagnosed cases of SSNHL were selected for this study. The mean age (in years) of cases was 47. Among 30 patients, 17(56.6\%) were males and remaining $13(43.3 \%)$ were female, with Male:Female ratio of 1.3:1 (Fig. 1). Demographic characteristics of the subjects were shown in Table I.

The average number of days from the onset of Table I: Demographic characteristics

\begin{tabular}{|c|c|}
\hline AGE & \\
\hline Mean & 47 \\
\hline Range & $25-70$ \\
\hline SEX & \\
\hline Male & 17 \\
\hline Female & 13 \\
\hline EAR & 12 \\
\hline Right & 18 \\
\hline Left & 14 \\
\hline $\begin{array}{c}\text { Days of onset to initiation of } \\
\text { ITS(mean) }\end{array}$ & \\
\hline
\end{tabular}




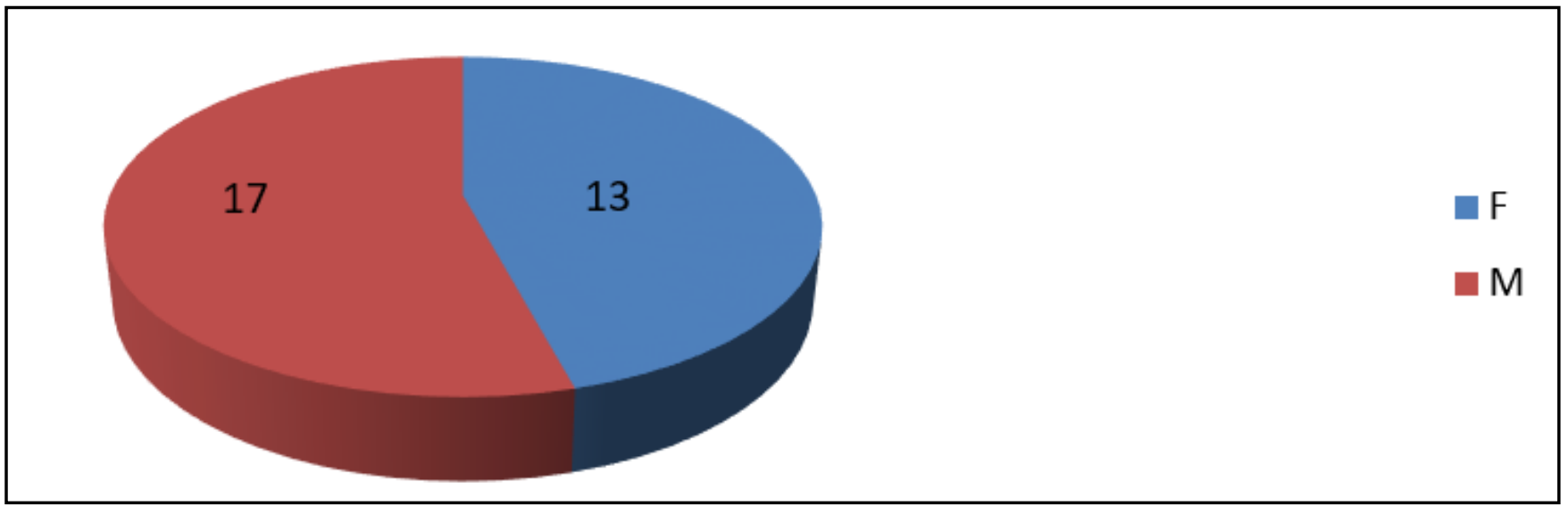

Fig. 1. Distribution of patients by sex $(\mathrm{N}=30)$

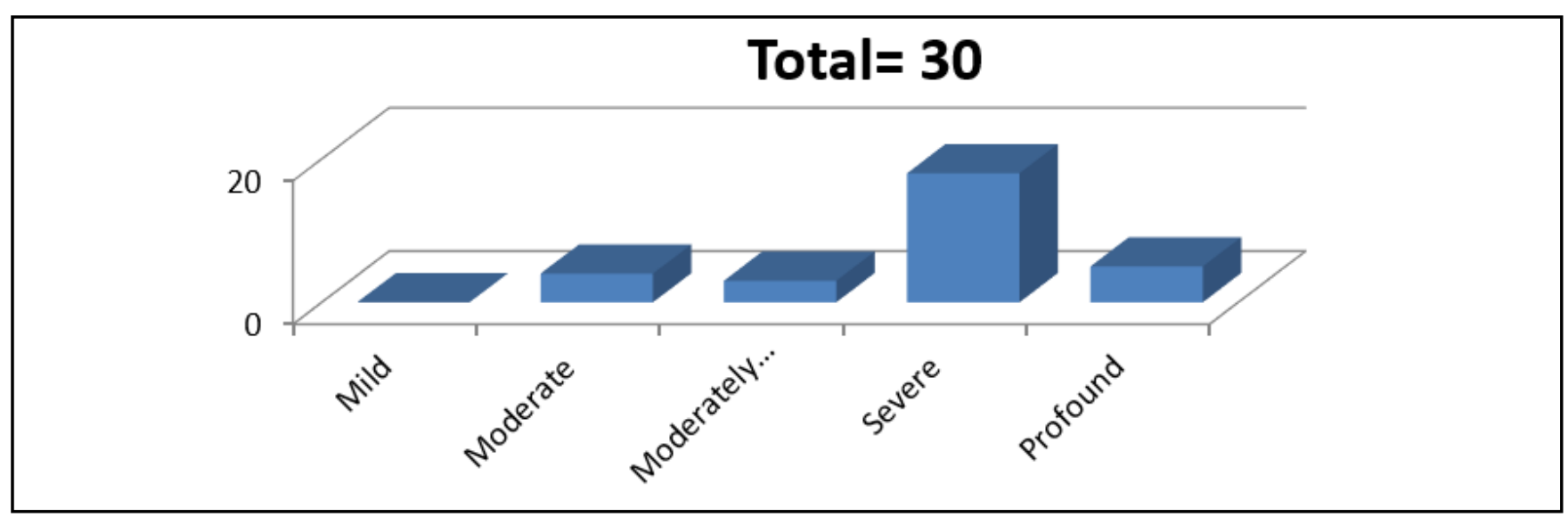

Fig. 2. Distribution of patients by degree of hearing loss

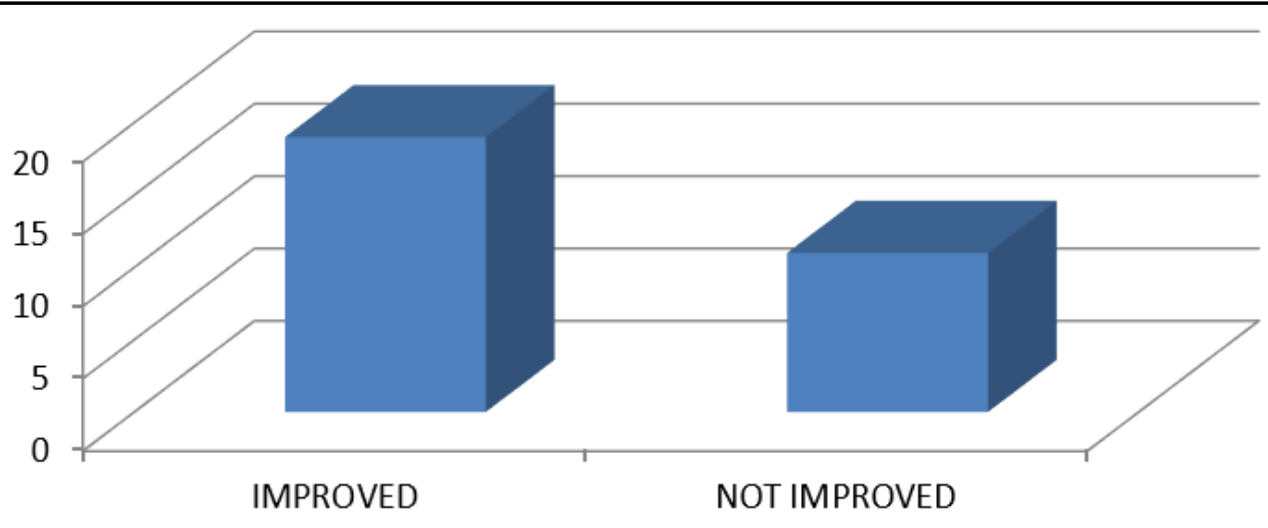

Fig. 3. Overall response $\operatorname{rate}(\mathrm{N}=30)$ 
hearing loss to the initiation of ITS was 14 days. The degree of hearing loss on presentation among the study subjects has been depicted in Fig. 2. With intratympanic steroid, $3(10 \%)$ patients having moderate, $1(3.3 \%)$ having moderate to severe, $13(43.3 \%)$ having severe and $2(6.6 \%)$ having profound hearing loss shows improvement. The average pure tone audiometry (PTA) was $76.6 \pm 14.7 \mathrm{~dB}$ and was improved to $45.6 \pm 16.7 \mathrm{~dB}$ in the last visit. The overall response rate was $63.3 \%$ $(19 / 30)$ as presented in Fig. 3. and was significant with $\mathrm{P}$ - value $(<0.05)$.

\section{Discussion}

Idiopathic sudden sensorineural hearing loss appears to be a true otologic emergency, with a time window of no more than two to four weeks to administer therapy. It is widely appreciated that the chance of treatment benefit decreases with increasing time from hearing loss onset to initiation of therapy. ${ }^{10}$

Today steroid therapy is considered to be the most effective and common method for SSNHL treatment. At the same time a huge number of side effects appears to be associated with the long term systemic use of steroids. Furthermore, a low dose of systemic steroids cannot create an ideal therapeutic effect because of their limited capacity to penetrate through the hematoperilymphatic barrier. ${ }^{11}$

In our study, nearly $63 \%$ patients showed improvement in hearing with intratympanic administration of dexamethasone after unsuccessful oral steroid administration. Swamy et al. conducted prospective study on 40 patients to study the effect of intratympanic steroid in patients with SSNHL and found significant improvement of about 50\% in hearing loss. ${ }^{7}$

In our study the average day of treatment initiation with intratympanic steroid from the onset of hearing loss was two weeks. Zhao et al. claim that the treatment which was started within two weeks after the onset of hearing loss is more effective than later. ${ }^{12}$ Banerjee and Parnes demonstrated a significantly better hearing improvement in patients treated with intratympanic steroid within 10 days of SSNHL onset, compared with those treated after 10 days. ${ }^{13}$
In our study we used $0.5-1 \mathrm{ml}$ of injection dexamethasone intratympanically $(4 \mathrm{mg} / \mathrm{ml})$ once weekly for three weeks. Till now there is no fixed treatment protocol of intratympanic dexamethasone for SSNHL. Liu rong et al. performed a study on 71 patients to evaluate the different protocols of intratympanic dexamethasone injection in patients with SSNHL who have failed to respond to typical medical treatment. Four injection protocols were employed: injection of $0.3 \mathrm{ml}$ dexamethasone $(5 \mathrm{mg} / \mathrm{ml})$ three times a week for 3 weeks for a total dose of $13.5 \mathrm{mg}$ (Group I, $\mathrm{n}=16$ ); injection of $0.6 \mathrm{ml}(5 \mathrm{mg} / \mathrm{ml})$ dexamethasone three times a week for 3 weeks for a total dose of $27 \mathrm{mg}$ (Group II, $\mathrm{n}=18)$; injection at $0.3 \mathrm{ml}(5 \mathrm{mg} / \mathrm{ml})$ week for 6 weeks for a total dose of $9 \mathrm{mg}$ (Group III, $\mathrm{n}=18$ ); injection at $0.3 \mathrm{ml}(5 \mathrm{mg} / \mathrm{ml}) / 2$ days for a total dose of $4.5 \mathrm{mg}$ (Group IV $\mathrm{n}=19$ ). The total effective rate was $37.5 \%, 38.89 \%, 33.33 \%$ and $36.84 \%$ for each treatment protocol, respectively, with no statistical difference between them $(\mathrm{P}>0.05)$. The results suggest that a total dose of $4.5 \mathrm{mg}$ (injected once a week for 3 weeks) is the most adequate protocol, and increasing dose or injection frequency yields no additional benefit. ${ }^{14}$

Belhassen et al. studied the efficacy of intratympanic steroid injection as a salvage option in sudden SNHL treatment. ${ }^{4}$ Their data support intratympanic steroid injections are effective in the treatment of sudden SNHL for moderate and severe hearing loss. Our study reports that intratympanic dexamethasone injection are also effective in the treatment of sudden SNHL for moderate to severe degree of hearing loss.

Without treatment, the spontaneous recovery rate is 30-60 per cent, mostly within the first two weeks of hearing loss. The degree of hearing loss and the interval before commencing treatment are the two main factors influencing prognosis. A longer interval of time before starting therapy is also associated with a lower recovery rate. Other factors linked to poorer prognosis have been postulated, such as the presence of vertigo. Many researchers have reported that intratympanic steroid injection provides higher perilymph concentrations in the inner ear with minimal systemic toxicity. In fact, the efficacy of intratympanic steroids as salvage therapy for patients who fail to respond to high-dose systemic corticosteroids has been an interesting option. Some 
authors have proposed the use of intratympanic injection as first line therapy in all cases of sudden sensori-neural hearing loss. ${ }^{15}$

\section{Conclusion}

This study shows that, intratympanic dexamethasone injection significantly improves the prognosis of ISSNHL that has failed on oral steroid, and is an effective and safe therapy. Further randomized studies and meta-analysis are recommended to address the current controversy regarding standardization of protocol for the treatment of sudden sensori-neural hearing loss.

\section{References}

1. Tony N, Catherine R. Idiopathic sudden sensorineural hearing loss. In: John CW, Raymond WC, editors. Scott-Brown's Otorhinolaryngology, Head and Neck Surgery. Eighth ed, Taylor \& Francis Group, LLC, 2018, 739-47

2. Anyah A, Mistry D, Kevern E, et al. Idiopathic Sudden Sensorineural Hearing Loss: Average Time Elapsed Before Presentation to the Otolaryngologist and Effectiveness of Oral and/or Intratympanic Steroids in Late Presentations. Cureus 2017; 9(12): e1945. DOI 10.7759/cureus. 1945

3. Haynes DS, O'Malley M, Cohen S, Watford K, Labadie RF. Intratympanic Dexamethasone for Sudden Sensorineural Hearing Loss After Failure of Systemic Therapy. Laryngoscope 2007, 117:3-15

4. Belhassen S, Saliba I. Intratympanic Steroid injection as a salvage treatment for Sudden Sensorineural Hearing Loss. J Laryngol Otol. 2014; 1-6.
5. Wilson WR, Byl FM, Laird N. The efficacy of steroids in the treatment of idiopathic sudden hearing loss. A doubleblind clinical study. Archives of Otolaryngology 1980;106(12):772-6

6. Chen WT, Lee JW, Yuan CH, Chen RF. Oral steroid treatment for idiopathic sudden sensorineural hearing loss. Saudi Med J. 2015; 36(3):291-6

7. Swamy KM, Arati G. Effect of intratympanic dexamethasone injection in sudden idiopathic sensorineural hearing loss. International Journal of Otorhinolaryngology and Head and Neck Surgery 2016 Oct; 2(4):258-62

8. Hyun JS. Intratympanic Steroid Injection in Tinnitus Management. Hanyang Med Rev. 2016; 36:125-30

9. Marx M, Younes E, Chandrasekhar SS, Ito J, et al. International consensus (ICON) on treatment of sudden sensorineural hearing loss. European Annals of Otorhinolaryngology, Head and Neck diseases 2018; 135: S23-S28

10. Steven DR, Robert D, Karen JD. Idiopathic sudden sensorineural hearing loss. In: Ashley W, James BS, editors. Ballengers Otorhinolaryngology: Head and neck surgery.18th ed. People's medical publishing house-USA, 2016, 1035-47

11. Sergey K, Arusyak A, Alexandr G, Edgar A, Alexandra K. Intratympanic Steroids for Sudden Sensorineural Hearing Loss. Int. Adv. Otol. 2011; 7:(3) 323-32

12. Zhao H, Zhang TY, Jing JH, Fu YY, Luo JN. Prognostic factors for patients with the idiopathic sudden sensorineural hearing loss [in Chineese]. Zhonghua Er Bi Yan Hou Tou Jing Wai Ke Za Zhi. 2008; 43:660-4

13. Banerjee A, Parnes LS. Intratympanic corticosteroids for sudden idiopathic sensorineural hearing loss. Otol Neurotol 2005;26:878-81

14. Rong-rong L, Ji-chuan C, Changyou J. Sudden sensorineural hearing loss treated with intratympanic dexamethasone injection. Journal of Otology 2010; 5(1):4-7

15. Rauch SD. Intratympanic steroids for sensorineural hearing loss. Otolaryngol Clin North Am. 2004; 37:1061-74. 